\title{
Editorial
}

Caries Research

Published online: October 18, 2017

DOI: $10.1159 / 000480305$

\section{Consensus Statements}

Clinicians know what they are saying and are completely understood by other clinicians, or so it should be. The problem is that the same words or phrases mean different things to different individuals. Similarly, how clinical conditions are treated may be determined by the knowledge of the practitioner which may not necessarily be complete or adequate. To overcome these potential deficiencies, learned societies or governments develop consensus statements which express evidenced-based, and state-of-the-art, knowledge. The methods used to produce such important documents are varied but the consensus methods commonly adopted in medical, dental, and health services research are the Delphi process, the nominal group technique (also known as the expert panel), and consensus development conferences. The consensus methods vary in how many questions they may be able to handle: only a few questions (4-6) tend to be considered in consensus development conferences, whereas the Delphi method and the nominal group technique can address a very large number of questions.

The main purpose of consensus statements is to provide the practitioner with the optimal and acceptable method to diagnose and treat certain diseases or injuries. However, consensus statements differ from medical/dental guidelines, another form of state-of-the-science public statements, medical/dental guidelines. According to the $\mathrm{NIH}$, "consensus statements synthesize new information, largely from recent or on-going medical/dental research, that has implications for re-evaluation of routine medical practices. They do not give specific algorithms or guidelines for practice."

\section{KARGER}

๑ 2017 S. Karger AG, Basel
There are many ways of producing medical/dental consensus, but the most usual way is to convene an independent panel of experts, either by a learned association or by a governmental authority. A consensus statement is developed by an independent panel of experts, usually multidisciplinary, convened to review the research literature for the purpose of advancing the understanding of an issue, procedure, or method. To avoid bias in the development of consensus statements complete transparency of methodology is essential. The involvement of funders, or lack of external funding, must be explicitly stated. The role of the experts is vital, and any conflicts of interest, commercial or intellectual, they might individually have must be revealed as these conflicts might influence decision-making. Intellectual conflicts of interest may be defined as "academic activities that create the potential for an attachment to a specific point of view that could unduly affect an individual's judgment about a specific recommendation." Thus, a patent holder or developer of a diagnostic method might not be an appropriate member of the expert panel. However, complete transparency might ameliorate these conflicts since these individuals may be experts held in high scientific esteem and valuable members of the expert panel. It cannot be emphasized too strongly that at all stages of producing a consensus statement the independence of the experts is important. Similarly, the voting of each expert for or against consensus must be undertaken so that the experts are not subject to external pressures to influence his/her decision. Voting should be anonymous. Unless each expert at the time of voting 
is free of commercial or intellectual conflicts of interest of other experts, then the outcome of the vote may not be independent.

Consensus does not have to be $100 \%$. A lower level of agreement may be used and taken as "consensus" but this should be decided prior to the process and the level of agreement that will be considered "consensus." Since consensus statements provide a "snapshot in time" of the state of knowledge in a particular topic, they must periodically be re-evaluated and published again, replacing the previous consensus statement.

Transparency of the process is essential and AGREE II [http://www.agreetrust.org/resource-centre/agree-reporting-checklist/] is a 23-item checklist comprising 6 quality domains that help "guidelines developers to follow a structured and rigorous development methodology, to conduct an internal assessment to ensure that their guidance is sound, or to evaluate guidelines from other groups for potential adaptation to their own context." AGREE II is a reliable tool widely used in the assessment of methodological rigor and transparency.

Caries Research will publish consensus statements produced using these protocols but essential information that must be reported includes:

- Scientific/clinical organization under whose auspices the process is initiated
- Method used to define the initial questions or statement

- Method used to select experts/participants

- Experience of individual experts/participants and potential conflicts of interest of each expert/participant

- Status and reporting of relevant systematic reviews

- Method used to achieve consensus; the process to obtain opinions of participants must be secret and confidential

- Clear definition of target "acceptable" level of consensus

- Level of consensus of individual responses or consensus statements

- The validity of the consensus decisions and the method(s) used to assess validity

- The impact of the consensus decisions on clinical practice and patient health

- The impact of the consensus decisions on future research

In conclusion, it is not the purpose of ORCA to dictate the method to be used to arrive at consensus. However, it must be remembered that the quality of the consensus is directly related to the unbiased application of predetermined detailed protocols with particular emphasis on the selection of experts/participants and on the method used to assess the level of consensus.

David Beighton, Editor-in-Chief 